\title{
ON THE BOUNDARY BEHAVIOR OF FUNCTIONS FOR WHICH THE RIEMANN IMAGE HAS FINITE SPHERICAL AREA
}

\author{
Dedicated to Mitsuru Ozawa on the Occasion of his Sixtieth Birthday
}

\author{
By James A. Jenkins and Kôtaro Oikawa
}

$\S 1$. In 1912 Montel [10] proved a result which may be formulated as follows. Let $f(z), z=x+i y$, be regular and bounded in a half-strip : $a<x<b, y>0$ and let $f(\xi+i y)$ have a limit as $y$ tends to infinity for a fixed $\xi, a<\xi<b$. Then $f(x+i y)$ has the same limit as $y$ tends to infinity for each $x$ in $(a, b)$, uniformly on any closed subinterval of $(a, b)$. In 1915 Lindelöf [9] showed that the same result follows from the assumption that the limit exists on a more general path. Hardy, Ingham and Pólya [5] considered in the formulation of Montel the problem for $|f(x+i y)|$ rather than $f(x+i y)$ and found that the existence of the limit for the modulus for one $\xi$ was not enough but that if $\lim _{y \rightarrow \infty}|f(x+i y)|$ existed for $x=\alpha, \beta$ with $a<\alpha<\beta<b$ and $\beta-\alpha<(b-a) / 2$ conclusions analogous to Montel's are obtained. They gave also some extensions and embellishments were made by Miss Cartwright [2], Hayman [6] and Bowen [1]. We will study a similar problem for the class of functions such that the Riemann image given by the mapping has finite spherical area and will find that then the existence of the limit for the modulus on one line implies a situation analogous to Montel's result. It turns out that results of this sort can be most appropriately formulated in terms of cluster sets. Also we can substantially weaken the requirements on the set of approach. Finally even in the family of bounded functions the cluster set interpretation provides new insights.

§2. Our results are most conveniently stated in terms of a half-strip for approach to its boundary point at infinity so we indicate briefly our terminology.

DEFINITION 1. Let $S$ denote the half-strip $a<x<b, y>0$. Let $T$ be a subset of $S, T_{\lambda}$ its subset on which $y \geqq \lambda$. For $f$ defined on $S$ the cluster set $C(f, T, \sigma)$ of $f$ on $T$ at $\sigma$, the boundary point of $S$ at the point at infinity, is defined to be $\bigcap_{\lambda>0} \mathrm{Cl} f\left(T_{\lambda}\right)$ where $\mathrm{Cl}$ denotes closure on the sphere.

DEFINITION 2. $\mathscr{F}(S)$ denotes the family of functions meromorphic on $S$ for

Research supported in part by the National Science Foundation

Received September 21, 1984 
which the Riemann image of $S$ has finite spherical area.

THEOREM 1. Let $f \in \mathscr{I}(S)$. Let $T$ be a closed subset of $S$ such that for fixed $L(>0)$ every rectangle $a<x<b, Y \leqq y \leqq Y+L, Y>0$, contains a subcontinuum of $T$ of diameter at least $\delta$ for a certain positive $\delta$. Let $U$ be a subset of $a^{\prime} \leqq x \leqq b^{\prime}$, $y>0$ with $a<a^{\prime}<b^{\prime}<b$. Then $C(f, U, \sigma) \subset C\left((f, T, \sigma)\right.$. For $x$ in $\left[a^{\prime}, b^{\prime}\right]$ the spherical distance of $f(x+i y)$ from $C(f, T, \sigma)$ tends to zero uniformly as $y$ tends to infinity.

We begin by proving the following auxiliary result.

LEMMA. Let $K$ be a continuum of diameter at least $\delta(>0)$ in the rectangle $R: a \leqq x \leqq b .-(1 / 2) L \leqq y \leqq(1 / 2) L$ and let $P$ be $a$ point $(\notin K)$ on the segment $a+\eta$ $\leqq x \leqq b-\theta, y=0, \eta, \theta>0, \eta+\theta<b-a$. Let $\Gamma$ be the family of open locally rectifiable arcs in $R-\partial R-\{P\}-K(\partial R$ denotes the boundary of $R)$ tending to $K$ in each sense and separating $P$ from $\partial R-K$ in $R-\partial R-K$. Then the module $m$ of $\Gamma$ is bounded from zero by a quantity depending only on $b-a, L, \delta, \eta, \theta$.

Evidently, we may assume without loss of generality that $\theta=\eta, \delta<(1 / 2) \eta$, $\eta<(1 / 2) L$. Suppose that $K$ meets one of the sets $a \leqq x \leqq a+(1 / 2) \eta,-(1 / 2) L \leqq y \leqq$ $(1 / 2) L ; b-(1 / 2) \eta \leqq x \leqq b,-(1 / 2) L \leqq y \leqq(1 / 2) L ; a \leqq x \leqq b,(1 / 2) L-(1 / 2) \eta \leqq y \leqq(1 / 2) L$; $a \leqq x \leqq b,-(1 / 2) L \leqq y \leqq-(1 / 2) L+(1 / 2) \eta$, for example the first one. Let $a=$ $\min _{z \in K} \mathcal{R} z$ and let $\hat{R}$ be the rectangle $\hat{a} \leqq x \leqq b,-(1 / 2) L \leqq y \leqq(1 / 2) L$. Further let $\hat{m}$ be the module related to $\hat{R}$ as $m$ is to $R$. Evidently $\hat{m} \leqq m$. Moreover $\hat{m}$ is equal to the triad module $[7,8] m(P, \alpha, D)$ where $D$ is the component of $\hat{R}-K$ containing $P$ and $\alpha$ is the open border arc of $D$ determined by $K$. Let $\tilde{m}$ be the triad module $m(P, \beta, D)$ where $\beta$ is the open border arc of $D$ complementary to $\alpha$. It is well known (and very easy to prove) that $\hat{m} \tilde{m}=4$. Also the Euclidean metric provides the upper bound $L(b-\hat{a}) / \delta^{2}$ for $\tilde{m}$. Thus $\hat{m} \geqq 4 \delta^{2} L^{-1}(b-a)^{-1}$. The same bound applies similarly in the other cases. Otherwise let $Q$ be a point of $K$ closest to $P$. A circle with centre $Q$ of radius $(1 / 2) \delta$ must meet $K$. The locus $\Lambda$ of points at a constant distance $(1 / 2) \delta$ from the segment $s$ joining $P$ and $Q$ lies in $R-\partial R$ and the module of $\Gamma$ is at least as large as the module of the doubly-connected domain bounded by $\Lambda$ and $s$. Again the latter quantity is bounded from zero by a value of the desired sort.

Now let $\left\{z_{n}\right\}$ be a sequence of points in $U-T$ tending to $\sigma$ and let $C$ be a continuum such that $C(f, T, \sigma) \subset \mathcal{C}$. For $\nu>0$ let $\mathcal{C}_{\nu}$ be the set of points at spherical distance at most $\nu$ from $\mathcal{C}$. It is a continuum. Suppose that $\left\{f\left(z_{n}\right)\right\}$ has an accumulation point $B$ at distance $2 \mu$ from $\mathcal{C}(\mu>0)$. Let $\Delta$ be the complementary component of $\mathcal{C}_{\mu}$ containing $B$. ( $\Delta$ is simply-connected.) Let $S_{Y}^{\prime}$ denote the set $a<x<b, y>Y$. Let $e$ be prescribed with $0<e<\mu$. For $Y$ sufficiently large the Riemann image of $S_{Y}^{\prime}$ under $f$ will have spherical area less than $e$, that image will fail to cover some point of $\Delta$ which we may assume to be the point at infinity and $f\left(T_{Y}\right) \subset \mathcal{C}_{e}$. There will be a subsequence $\left\{z_{n_{k}}\right\}$ of $\left\{z_{n}\right\}$ with 
$\mathcal{I} z_{n_{k}}>Y+(1 / 2) L$ and $f\left(z_{n_{k}}\right) \in \Delta$, all $k$. The rectangle $a \leqq x \leqq b, \quad g z_{n_{k}}-(1 / 2) L \leqq y$ $\leqq \mathfrak{I} z_{n_{k}}+(1 / 2) L$ contains a continuum $K_{k}$ in $T$ of diameter at least $\delta$. $f\left(z_{n_{k}}\right)$ can be joined to the point at infinity by a path $\tau_{k}$ in $\Delta$. Let $\Gamma_{k}$ be the family of open locally rectifiable arcs in $S_{Y}^{\prime}-\left\{z_{n_{k}}\right\}$ tending in each sense to $K_{k}$ and separating $z_{n_{k}}$ from $\partial S_{Y}^{\prime}-K_{k}$. If $\gamma \in I_{k}^{\prime}$ it follows from the argument principle that $f(\gamma)$ meets $\tau_{k}$. Thus the spherical length of $f(\gamma)$ is at least $2(\mu-e)$. The metric induced in $S_{Y}^{\prime}$ by the spherical metric gives an upper bound for the module of $\Gamma_{k}$ as $e(2(\mu-e))^{-2}$. This, however, contradicts the result of the Lemma. Thus any accumulation point of $\left\{f\left(z_{n}\right)\right\}$ lies in $\mathcal{C}$ and $C(f, U, \sigma) \subset \mathcal{C}$. On the other hand for any point $H$ not in $C(f, T, \sigma)$ there is a continuum containing $C(f, T, \sigma)$ and not containing $H$. Thus $C(f, U, \sigma) \subset C(f, T, \sigma)$. The uniformity statement is immediate.

§3. The following corollaries serve to illustrate simple cases of Theorem 1 . Some of them might also be obtained by other techniques and admit considerably simplified direct proofs by the method of the extremal metric.

COROLlaRy 1. Let $f \in \mathscr{F}(S)$. Then for each half-line $L_{x}: a<x<b, y>0$ the cluster set $C\left(f, L_{x}, \sigma\right)$ is the same.

This result is known for univalent functions [3, p. 178; 12, p. 275].

Corollary 2. Let $f \in \mathscr{F}(S)$. Let $\lim _{y \rightarrow \infty}|f(\xi+i y)|$ exist for a certain value $\xi$, $a<\xi<b$, and have the value $A$. Then $\lim _{y \rightarrow \infty}|f(x+i y)|=A$ for each $x$ in $a<x<b$, uniformly on any closed subinterval.

COROllary 3. Let $f \in \mathscr{F}(S)$. Let $\lim _{z \rightarrow \sigma} f(z)$ exist on a path in $S$ tending to $\sigma$. Then $\lim _{z \rightarrow \sigma} f(z)$ exists on any path in $S$ tending non-tangentially to $\sigma$ and has the same value.

COROLlary 4. Let $f \in \mathscr{F}(S)$. Let $\lim _{z \rightarrow \sigma} R f(z)=c$ on a path in $S$ tending to $\sigma$ and $\lim _{z \rightarrow \sigma} g f(z)=d$ on a path in $S$ tending to $\sigma$, where $c, d$ are real numbers. Then on any path in $S$ tending non-tangentially to $\sigma$ either $\lim _{z \rightarrow \sigma} f(z)$ exists and has the value $c+i d$ or $f(z)$ tends to the point at infinity. In either case the limit exists uniformly on the substrip for which $a^{\prime} \leqq x \leqq b^{\prime}, a<a^{\prime}<b^{\prime}<b$.

This is an analogue of a result due to Gehring and Lohwater [4] for bounded functions. It is readily seen that both alternatives are possible.

Remark. Evidently all these results extend to the case where $f$ is quasiconformal (not necessarily $(1,1)$ ) rather than meromorphic. It may be noted that Vuorinen [13] has studied the extension of Lindelöf's theorem to quasiconformal 
mappings in higher dimensions.

§4. THEOREM 2. Let $f$ be regular and bounded in $S$ and let $\lim _{y \rightarrow \infty}|f(\alpha+i y)|$ $=\lim _{y \rightarrow \infty}|f(\beta+i y)|=A$ for $a<\alpha<\beta<b$. Suppose that $V=C\left(f, L_{\alpha}, \sigma\right) \cup C\left(f, L_{\beta}, \sigma\right)$ does not coincide with $\{|w|=A\}$. Then $\lim _{y \rightarrow \infty}|f(x+i y)|=A$ for every $x$ in $a<x<b$. uniformly on any closed subinterval.

Let $\hat{S}$ denote the set $\alpha<x<\beta, y>0$. It follows from a well-known theorem $[3$, p. $91 ; 11$, p. 17] that $\partial C(f, \hat{S}, \sigma) \subset V$, thus $C(f, \hat{S}, \sigma) \subset V$ since $f$ is bounded. In particular $C\left(f, L_{x}, \sigma\right) \subset\{|w|=A\}$ for every $x$ in $\alpha<x<\beta$. The theorem then follows from the original Hardy-Ingham-Pólya result.

\section{BIBLIOGRAPHY}

[1] N. A. Bowen, On the limit of the modulus of a bounded regular function, Proceedings of the Edinburgh Mathematical Society, Vol. 14, 1964-65, pp. 21-24.

[2] M. L. CARTwRIGHT, A generalization of Montel's theorem, Journal of the London Mathematical Society, Vol. 37, 1962, pp, 179-187.

[3] E.F. Collingwood and A. J. Lohwater, The Theory of Cluster Sets, Cambridge Tracts in Mathematics and Mathematical Physics, No. 56, Cambridge University Press, Cambridge, England, 1966.

[4] F.W. Gehring and A.J. Lohwater, On the Lindelöf theorem, Mathematische Nachrichten, Vol. 19, 1958, pp. 165-170.

[5] G. H. Hardy, A.E. Ingham and G. Pólya, Notes on moduli and mean values, Proceedings of the London Mathematical Society (2), Vol. 27. 1928, pp. 401409.

[6] W.K. HAYman, On the limits of moduli of analytic functions, Annales Polonici Mathematici, Vol. XII, 1962, pp. 143-150.

[7] James A. Jenkins, On the Denjoy conjecture, Canadian Journal of Mathematics, Vol. 10, 1958, pp. 627-631.

[8] James A. Jenkins, On the Phragmén-Lindelöf theorem, the Denjoy conjecture and related results, Mathematical Essays Dedicated to A.J. Macintyre, Ohio University Press, Athens, Ohio, 1970, pp. 183-200.

[9) E. LiNDELöF, Sur un principe général de l'analyse et ses applications à la théorie de la représentation conforme, Acta Societatis Scientiarum Fennicae, Vol. 46, 1915, pp. 1-35.

[10] P. Montel, Sur les familles de fonctions analytiques qui admettent des valeurs exceptionelles dans un domaine, Annales Scientifiques de l'École Normale Supérieure (3), Vol. 23, 1912, pp. 487-535.

[11] K. Noshiro, Cluster Sets, Ergebnisse der Mathematik und ihrer Grenzgebiete, Heft 28, Berlin-Göttingen-Heidelberg, 1960.

[12] M. Oнтsuka, Dirichlet Problem, Extremal Length and Prime Ends, Van Nostrand Reinhold Mathematical Series, New York, Cincinnati, Toronto, London, Melbourne, 1970. 
L13] M. VuORINEN, Lindelöf-type theorems for quasiconformal and quasiregular mappings, Complex Analysis, Banach Center Publications, Vol. 11, 1983, pp. 353-362.

Department of Mathematics

WASHINGTON UNIVERSITY

St. Louis MO 63130

U.S. A.
College of Arts and Sciexces

UNIVERSITY OF TOKYO

Komaba, Meguro, Tokyo,

JAPAN 